\title{
Pubertad y deportes atléticos en adolescentes femeninas
}

\author{
Panagiota (Nota) Klentrou \\ Departamento de Educación Física y Kinesiología, Universidad Brock, St. Catharines, Canadá
}

\author{
Palabras clave \\ Atletas femeninas, entrenamiento/crecimiento/ \\ maduración · Desarrollo hormonal · Menarquia • Función \\ reproductora $\cdot$ Composición corporal $\cdot$ Restricción \\ alimentaria $\cdot$ Amenorrea, atlética
}

\section{Extracto}

La creciente participación de niños pequeños en el entrenamiento físico intensivo, durante las últimas décadas, ha generado temores con respecto a sus efectos potenciales sobre el crecimiento y la maduración de los niños. La pubertad en el humano se caracteriza por cambios hormonales considerables, que resultan tanto en maduración física como en maduración sexual. Dado que un entrenamiento intensivo antes de la pubertad, junto a los efectos metabólicos potenciales del seguimiento de una dieta, puede alterar la función hipotalámica e hipofisaria, el momento en que se inicia el entrenamiento atlético ha sido implicado como un factor en el retraso de la menarquia y la maduración sexual en atletas femeninas. Por otra parte, en algunos estudios se ha dejado entrever la probabilidad de que el retraso de la menarquia puede deberse a factores genéticos. Las niñas que maduran más tardíamente, a menudo se autoseleccionan o son reclutadas por preparadores para deportes que favorecen cuerpos de tamaños reducidos o muy delgados. También se ha utilizado la composición corporal para explicar el retraso de la menarquia y las irregularidades menstruales que se observan en atletas de elite. Se ha descrito una mayor prevalencia de disfunción menstrual en atletas adolescentes que participan en deportes dependientes del peso, en comparación con lo que se observa en otros deportes. No obstante, tal como se ha dejado entrever recientemente, no existe una relación directa entre causa y efecto entre la obesidad y la reproducción y, en realidad, la función reproductora en las mujeres está regulada por la disponibilidad energética y no por la grasa corporal. Está justificado intensificar la investigación para indagar adicionalmente esta interacción entre los cambios a corto plazo en la disponibilidad de los combustibles y la amenorrea atlética en adolescentes femeninas. Se llega a la conclusión de que, teniendo en cuenta los numerosos factores que, según se ha comprobado, influyen sobre la menarquia y la menstruación, el papel desempeñado por el mero entrenamiento físico como factor causal del inicio más tardío de la pubertad y de las irregularidades menstruales en niñas físicamente activas sigue siendo dudoso. Es imprescindible una investigación basada en estudios diseñados longitudinalmente para identificar si las diferencias en la maduración, observadas entre atletas femeninas y niñas que no practican actividades atléticas, son el resultado de la naturaleza o la nutrición, y cuál es el equilibrio entre ambos factores. Copyright $\odot 2006$ Nestec Ltd., Vevey/S. Karger AG, Basel

\section{Introducción}

La pubertad es un acontecimiento significativo en el crecimiento y la maduración humana, por una parte porque se asocia a notables cambios fisiológicos y psicológicos y, por otra, porque se caracteriza por ajustes neuroendocrinos significativos. En consecuencia, duran-

\begin{tabular}{|c|c|}
\hline KARGER & $\begin{array}{l}\text { (c) } 2006 \text { Nestec Ltd., Vevey/S. Karger AG, Basel } \\
0252-8185 / 06 / 0642-0085 \$ 23.50 / 0\end{array}$ \\
\hline $\begin{array}{l}\text { Fax +41 } 613061234 \\
\text { E-Mail karger@karger.ch } \\
\text { www.karger.com }\end{array}$ & $\begin{array}{l}\text { Accesible online en: } \\
\text { www.karger.com/ans }\end{array}$ \\
\hline
\end{tabular}

Nota Klentrou, $\mathrm{PhD}$

Department of Physical Education and Kinesiology Brock University

St. Catharines, Ont. L6L 3M7 (Canada)

Tel. +1 905688 5550/ext. 4538, Fax +1 905688 8364, E-Mail nota.klentrou@brocku.ca 
te sus años puberales, el estado hormonal de una atleta adolescente pasa a través de una serie de adaptaciones inducidas por el desarrollo, siempre cambiantes y derivadas del entrenamiento.

El creciente número de adolescentes femeninas que compitieron en eventos deportivos durante las últimas décadas ha intensificado el interés por la fisiología de la niña que practica ejercicio. El crecimiento y la maduración se caracterizan por la variación individual y, aunque están sometidos a control genético y neuroendocrino, los factores ambientales, entre los que destacan el tipo, la intensidad y la duración del entrenamiento, pueden también ejercer alguna influencia [1]. Hasta la fecha, nuestro conocimiento del efecto que produce el entrenamiento deportivo sobre los niños en fase de crecimiento, especialmente las niñas, es limitado por la dificultad de distinguir entre los efectos del entrenamiento y los del crecimiento normal [1]. En consecuencia, el efecto del entrenamiento intensivo sobre el crecimiento y la maduración de niñas que participan en deportes competitivos genera acciones fisiológicas paralelas que interactúan con las implicaciones potenciales para el estado de salud y el desarrollo de la adolescente femenina que practica ejercicio, oponiéndose en ocasiones a las mismas. Está claro que la nutrición es también un factor importante debido al papel que ejerce tanto en la maduración física como en la función metabólica correcta durante el entrenamiento y el rendimiento.

En este artículo examinaremos la respuesta al entrenamiento intensivo antes y durante la pubertad en las niñas. Se demostrará que en atletas femeninas de elite, especialmente en las más jóvenes, la combinación de entrenamiento intensivo y nutrición inadecuada causa a menudo retraso de la menarquia e irregularidades menstruales que pueden dar lugar a complicaciones del estado de salud en una etapa posterior de la vida. La discusión se centrará adicionalmente en si las diferencias en la maduración observadas entre atletas femeninas jóvenes y las niñas que no practican actividades atléticas son consecuencia de la naturaleza, la nutrición o una combinación de ambas.

\section{Pubertad y menstruación}

El proceso normal del ciclo menstrual femenino está regulado por una serie de interacciones complejas de hormonas hipofisarias y ováricas. Entre estas hormonas destaca la hormona liberadora de gonadotopinas ( $\mathrm{GnRH})$, que segrega el hipotálamo y actúa sobre la hipófisis para liberar la hormona foliculoestimulante (FSH) y la hormona luteinizante $(\mathrm{LH})$. Durante la infancia, el hipotálamo es sensible a los niveles bajos de estrógeno, que ejercen un efecto de retroalimentación negativo inhibiendo la actividad hipotalámica. El desencadenante exacto de la pubertad y la menarquia no se conoce todavía a ciencia cierta; la aparición de acontecimientos relacionados, así como la cronología de la menarquia, varía considerablemente y es influido por la nutrición, la herencia, el estado de salud, el porcentaje de grasa corporal, la hormona del crecimiento y otros factores de crecimiento, así como la maduración del eje hipotalámico-hipofisario que, a su vez, depende de una multitud de factores [2-4]. En general, la pubertad se inicia en las mujeres cuando el hipotálamo madura y llega a ser menos sensible a la retroalimentación negativa, fomentando un incremento de la secreción de GnRH por las células neurosecretoras presentes en el hipotálamo que, a su vez, desencadenan el inicio de la secreción de las hormonas gonadotópicas LH y FSH en la hipófisis anterior. Cantidades suficientes de FSH y LH estimulan el desarrollo folicular en los ovarios, el desarrollo de características sexuales secundarias y un incremento de la liberación de esteroides sexuales femeninos (estrógeno y progesterona) a partir de los ovarios [2]. El estrógeno y la progesterona siguen incrementándose a lo largo de la pubertad y desarrollan un papel clave, tanto en la maduración física como en la maduración sexual [3]. En el curso de las fases del ciclo menstrual, la FSH fomenta el desarrollo del folículo y la secreción de estrógeno por los ovarios, mientras que la LH estimula la ovulación y la secreción de estrógeno y progesterona. El ciclo menstrual se inicia con la menstruación. En la fase folicular, en los días previos a la ovulación, los niveles de FSH y LH se elevan, con predominio estrogénico. Después de la ovulación, durante la fase luteínica del ciclo, la progesterona supera al estrógeno, dado que el útero se prepara para un embarazo potencial.

Las atletas se preocupan frecuentemente por el número de días de menstruación y el volumen del flujo menstrual. Un ciclo normal se suele describir por una duración media de 28 días aunque, en realidad, un ciclo de 28 días aparece únicamente en el $12,4 \%$ de todos los ciclos. En estudios sobre la duración de las menstruaciones regulares se han registrado variaciones en la duración del ciclo 'normal' de 21 a 35 días [2, 3]. La duración menstrual también varía considerablemente, dado que el flujo normal fluctúa entre 2 y 8 días en promedio $[2,3]$.

Se ha dado a entender que el ejercicio intensivo puede alterar el estrógeno (fundamentalmente el componente estradiol) y la progesterona. En estudios se ha demostrado que aparecen cambios fisiológicos, tanto en la fase folicular como en la fase luteínica, en presencia de un entrenamiento y un rendimiento aerobio o anaerobio [5]. 
Tabla 1. Edad en el momento de la menarquia (media \pm DE) en adolescentes atléticas y adolescentes ajenas a las actividades atléticas

\begin{tabular}{|c|c|c|c|}
\hline Estudio & País & Grupo deportivo & $\begin{array}{l}\text { Edad en el momento } \\
\text { de la menarquia, años }\end{array}$ \\
\hline Torstveit y Sundgot-Borgen [10], 2005 & Noruega & $\begin{array}{l}\text { Atletas femeninas de elite }(n=669) \\
\text { Controles de edades equiparables }(n=607)\end{array}$ & $\begin{array}{l}13,4 \pm 1,4^{*} \\
13,0 \pm 1,3\end{array}$ \\
\hline Klentrou y Plyley [11], 2003 & Canadá & $\begin{array}{l}\text { Gimnastas rítmicas }(n=30) \\
\text { Controles de edades equiparables }(n=40)\end{array}$ & $\begin{array}{l}13,6 \pm 1,2^{*} \\
12,3 \pm 0,8\end{array}$ \\
\hline Klentrou y Plyley [11], 2003 & Grecia & $\begin{array}{l}\text { Gimnastas rítmicas de elite }(\mathrm{n}=15) \\
\text { Controles de edades equiparables }(\mathrm{n}=38)\end{array}$ & $\begin{array}{l}14,2 \pm 0,3^{*} \\
12,8 \pm 0,9\end{array}$ \\
\hline Dusek [12], 2001 & Croacia & $\begin{array}{l}\text { Atletas entrenadas antes de la menarquia }(n=34) \\
\text { Atletas entrenadas después de la menarquia }(n=33) \\
\text { Controles de edades equiparables }(n=96)\end{array}$ & $\begin{array}{l}13,8 \pm 1,4^{*} \\
12,6 \pm 1,0 \\
13,0 \pm 1,2\end{array}$ \\
\hline Pigeon et al. [13], 1997 & Francia & $\begin{array}{l}\text { Bailarinas de ballet }(n=97) \\
\text { Controles }(n=30)\end{array}$ & $\begin{array}{l}13,5^{*} \\
12,0\end{array}$ \\
\hline Constantini y Warren [14], 1995 & Israel & $\begin{array}{l}\text { Nadadoras }(n=69) \\
\text { Controles }(n=279)\end{array}$ & $\begin{array}{l}13,8 \pm 0,2^{*} \\
13,0 \pm 0,1\end{array}$ \\
\hline Lindholm et al. [4], 1994 & Suecia & $\begin{array}{l}\text { Gimnastas artísticas }(\mathrm{n}=22) \\
\text { Controles }(\mathrm{n}=22)\end{array}$ & $\begin{array}{l}14,5 \pm 1,4^{*} \\
13,2 \pm 0,9\end{array}$ \\
\hline Malina et al. [15], 1994 & EE.UU. & Siete deportes - mezcla de atletas $(n=109)$ & $13,8 \pm 1,5$ \\
\hline Hata y Aoki [16], 1990 & Japón & $\begin{array}{l}\text { Atletas de elite }(\mathrm{n}=40) \\
\text { Atletas escolares }(\mathrm{n}=386) \\
\text { Atletas de escuela de segunda enseñanza }(\mathrm{n}=253)\end{array}$ & $\begin{array}{l}13,5 \pm 1,3 \\
12,9 \pm 1,2 \\
12,6 \pm 1,1\end{array}$ \\
\hline Calabrese [17], 1985 & EE.UU. & Gimnastas artísticas $(\mathrm{n}=20)$ & 14,2 \\
\hline Calabrese et al. [18], 1983 & EE.UU. & Bailarinas de ballet $(n=25)$ & 14,3 \\
\hline Malina [19], 1973 & EE.UU. & $\begin{array}{l}\text { Atletas de campo y pista }(n=66) \\
\text { Controles }(n=30)\end{array}$ & $\begin{array}{l}13,6 \pm 0,2^{*} \\
12,2 \pm 1,6\end{array}$ \\
\hline
\end{tabular}

${ }^{*} \mathrm{p} \leq 0,05$ (diferencia comunicada entre atletas y controles).

\section{Inicio de la pubertad en atletas femeninas}

La creciente participación de niñas pequeñas en el entrenamiento físico intensivo durante las últimas décadas ha suscitado temores en relación con sus efectos potenciales sobre el crecimiento, la maduración y la función reproductora de éstas [6]. Se ha observado que el entrenamiento intensivo retrasa el inicio de la pubertad en las niñas por alteración del desarrollo hormonal normal $[7,8]$. Se han descrito retrasos en el inicio del crecimiento puberal, retrasos en la edad del momento de la primera menarquia y retrasos en la maduración esquelética en niñas participantes en un entrenamiento atlético durante un mínimo de 15 horas por semana [9]. La mayoría de las discusiones sobre la influencia potencial del entrenamiento físico sobre la maduración sexual se han centrado en el incremento de la edad media en el momento de la primera me- narquia, que a menudo se describe en atletas femeninas adolescentes [4, 10-19]. En la tabla 1 se recopilan datos internacionales sobre la edad en el momento de la menarquia en atletas femeninas jóvenes, en comparación con controles que no practican actividades atléticas. No obstante, en algunos estudios se ha dejado entrever que el retraso de la menarquia se debe a factores genéticos, y que las niñas que maduran tardíamente a menudo se autoseleccionan para deportes que exigen cuerpos pequeños y una potencia muscular relativamente elevada, como es el caso de las gimnastas [20]. Según Kaprio et al. [21], se comprobó que el 37\% de la variancia en la edad de aparición de la menarquia era atribuible a factores genéticos aditivos. Además, la correlación entre los efectos genéticos aditivos en la edad de aparición de la menarquia y el índice de masa corporal (peso/talla ${ }^{2}$ ) era de 0,57 , lo que indica una proporción considerable de efectos genéticos. 
Los datos longitudinales limitados de niñas que participaban en deportes, en comparación con niñas no practicantes de actividades atléticas, indicaba la ausencia de efectos del entrenamiento sobre la cronología o el progreso de características sexuales secundarias, incluyendo el desarrollo de las mamas y el vello púbico [22].

Dado que el entrenamiento intensivo antes de la pubertad puede alterar la función hipotalámico-hipofisaria, se ha considerado que el momento del inicio del entrenamiento atlético es un factor que influye sobre el retraso de la menarquia. Las atletas femeninas que inician su entrenamiento en edades tempranas presentan frecuentemente un retraso de la menarquia [23]. Según Dusek [12], se ha descrito un retraso significativo de la menarquia en atletas croatas que iniciaron las actividades físicas antes del comienzo de la menstruación $(13,8 \pm 1,4$ frente a 12,6 $\pm 1,0$ años; $p \leq 0,001)$. También se ha recurrido a la composición corporal para explicar el retraso de la menstruación y las irregularidades de los ciclos menstruales en atletas de elite. Aún sin el respaldo de los criterios actuales, Frisch y McArthur [24] formularon la teoría de una asociación entre la regularidad menstrual y el nivel de grasa corporal, dando a entender que un nivel mínimo del $17 \%$ de grasa corporal es crucial para el inicio de la menstruación y el mantenimiento de un ciclo normal. Datos epidemiológicos recientes han demostrado que la edad en el momento de la menarquia era significativamente más tardía ( $\mathrm{p} \leq 0,001)$ en atletas que en controles $(13,4 \pm 1,4$ frente a 13,0 $\pm 1,3$ años, respectivamente), $y$ que difería entre los grupos deportivos, correspondiendo el mayor retraso a las atletas participantes en deportes relacionados con la 'delgadez' [10]. En consecuencia, entre las actividades preocupantes destacan las actividades estéticas y de delgadez como la gimnasia, el patinaje artístico, la danza, así como deportes en los que el control del peso es una estrategia de rendimiento integrada, y cualquier deporte en el cual el inicio del entrenamiento físico intensivo tiene lugar en una edad muy temprana.

En trabajos publicados se describe que las magnitudes del entrenamiento semanal de gimnastas artísticas jóvenes, prepuberales y/o al inicio de la pubertad, llegan a ser de hasta 36 horas por semana [25]. En consecuencia, no es sorprendente que algunos autores hayan comunicado que gimnastas femeninas de elite conllevan un riesgo de retraso de la maduración [23, 26, 27]. En gimnastas artísticas se ha descrito una reducción del potencial de crecimiento y un retraso de la menarquia $[4,8,26]$. No obstante, existe un importante debate sobre si el potencial de crecimiento de gimnastas artísticas de elite es influido por el entrenamiento intenso, que frecuentemente se inicia antes de la pubertad. Los datos longitudinales de gim- nastas femeninas que abarcan la infancia tardía y la adolescencia son limitados. Los resultados de dos estudios longitudinales, a corto plazo, han dado a entender que las gimnastas progresan hacia la pubertad con un inicio del crecimiento puberal menos intenso [26, 27]. En otros estudios se ha dejado entrever que este retraso no es atribuible al entrenamiento gimnástico de por si, sino más bien a factores genéticos o a ingestas calóricas insuficientes para satisfacer las demandas de energía del crecimiento y el entrenamiento físico $[28,29]$. Aunque en un estudio en gimnastas de edades comprendidas entre 7 y 10 años se comprobó una reducción del índice de masa corporal, se ha dado a entender que la diferencia era el resultado de la selección de atletas delgadas [30]. Este dato está respaldado también por un estudio reciente efectuado por Damsgaard et al. [31], basado en datos longitudinales, en el que se indica que las diferencias individuales en el tamaño corporal entre los participantes en deportes se manifestaba ya a una edad temprana ( 2 a 4 años), lo que permite suponer una influencia genética. En un estudio muy reciente sobre el inicio del crecimiento y la edad esquelética en la adolescencia de gimnastas femeninas de $8,7 \pm 1,5$ a $15,5 \pm 1,5$ años, se observó que las gimnastas presentan inicios adolescentes de la talla, la longitud estimada de las piernas y la talla en posición sedente que aparecen aproximadamente un año después y son algo menos intensas que en las niñas que no practican actividades atléticas [9]. La edad en el momento de la menarquia y la edad esquelética eran coherentes con una maduración somática más tardía. Por otra parte, el inicio del crecimiento adolescente de estas gimnastas belgas eran similares a las de otras muestras de niñas de tallas bajas, normales y de maduración tardía y los de niñas de maduración tardía con padres bajos [9]. En general, la literatura demuestra que aunque, en promedio, las atletas femeninas jóvenes que practican la mayoría de los deportes (es decir, natación, remo, baloncesto, voleibol y tenis) presentan tamaños corporales que igualan o superan las medianas de referencia de la población normal. Las gimnastas, las patinadoras artísticas y las bailarinas de ballet son más bajas y presentan uniformemente una masa corporal más liviana; sin embargo, las gimnastas tienen también padres más bajos que el promedio [1].

En actividades estéticas, como la gimnasia rítmica, el entrenamiento intensivo se inicia habitualmente mucho tiempo antes de la menarquia y el éxito está muy influido por el atractivo visual y la estética corporal. Ya a los 9 años de edad, a las gimnastas rítmicas se les exige satisfacer metas de peso poco realistas con objeto de conseguir o mantener un físico favorable. Se ha observado que el retraso de la menarquia, las irregularidades menstruales y 
la grasa corporal escasa son corrientes en las gimnastas rítmicas de elite, en cuyo contexto las que se encuentran en la fase premenárquica presentan una mayor frecuencia y duración de entrenamientos, así como un menor índice de masa corporal y de grasa corporal relativa, en comparación con las de la fase menárquica [11]. Además, el desarrollo de las mamas se retrasaba en las bailarinas de ballet, que mostraban un desarrollo escaso o nulo (estadio 1 o 2 de Tanner) a los 13 años de edad, en comparación con niñas normales que alcanzaban habitualmente el estadio 4 en el momento de la menarquia [32]. En gimnastas canadienses adolescentes, de elite, se observó un retraso de la menarquia y el desarrollo de las mamas en comparación con controles de edades equiparables [33]. En este estudio, el grupo mixto de gimnastas artísticas y rítmicas adolescentes presentaba pesos corporales y valores de grasa corporal relativa significativamente menores en comparación con las participantes de control normales. En consecuencia, el desarrollo de las mamas se retrasaba en las gimnastas en comparación con los controles (estadios 2,7 $\pm 0,2$ frente a 3,8 $\pm 0,1$ ), si bien la diferencia entre los grupos en el desarrollo del vello púbico no alcanzaba significación (estadios $3,1 \pm 0,2$ frente a $4,0 \pm 0,2)$.

También se ha formulado la hipótesis de que cuando la pubertad es interrumpida transitoriamente por el entrenamiento físico, la edad de maduración esquelética se estanca frecuentemente a medida que el crecimiento se retrasa y los esteroides gonadales descienden hasta niveles bajos $[7,8]$. Desafortunadamente, sólo un número muy limitado de estudios ha proporcionado alguna información sobre la tasa de excreción de esteroides gonadales y/o suprarrenales en niñas peripuberales activas. Malina et al. [34] demostraron que la actividad de los ejes hipofisario-suprarrenal e hipofisario-gonadal podría depender de la naturaleza de la actividad. Con esta finalidad, Peltenburg et al. [35] comunicaron que las gimnastas prepuberales presentaban niveles menores de estrona, testosterona y androstendiona que nadadoras de edades equiparables, mientras que los niveles circulantes de $17 \beta$ estradiol, dehidroepiandrosterona sulfato (DHEA-S), LH y FSH fueron similares entre los grupos atléticos. Por el contrario, los niveles de todas estas hormonas no difirieron entre las gimnastas y las nadadoras al comienzo de la pubertad [35]. En una muestra de 56 gimnastas premenárquicas (que entrenaban de 15 a 22 horas por semana), en comparación con 53 controles de edades equiparables, no se hallaron diferencias en los niveles urinarios de testosterona, cortisol y DHEA-S, mientras que la androstendiona urinaria era significativamente menor en las gimnastas que en los controles [36]. Tal como era de prever, en el estudio de Klentrou et al. [33], los niveles plasmáticos de $17 \beta$-estradiol y progesterona de las gimnastas premenárquicas, cuya menarquia se había retrasado, fueron similares a los determinados en el grupo de control durante la fase folicular, que a su vez fueron significativamente menores ( $p \leq 0,05)$ que los registrados en los controles durante la fase luteínica.

\section{Entrenamiento atlético durante la adolescencia}

A medida que mayor número de niñas llega a participar en un entrenamiento atlético intensivo a una edad temprana, los problemas de salud que se centran en la tríada atlética femenina se han convertido en una preocupación. En ciertas actividades, especialmente los deportes estéticos y los deportes de alta potencia para la masa corporal, un incremento del peso corporal puede influir negativamente sobre el rendimiento [37]. En consecuencia, la vida de una atleta adolescente comprometida con estas actividades implica frecuentemente una combinación de entrenamiento físico intensivo y presión para alcanzar metas de peso poco realistas, como medio para mejorar el rendimiento y/o el atractivo visual. En consecuencia, el intento de controlar el peso y/o conseguir un físico deseable es a menudo responsable de trastornos del comer que se han asociado a retrasos en el crecimiento y la maduración sexual en atletas adolescentes [38]. Los trastornos del comer, la disfunción menstrual y la osteoporosis representan los tres componentes interrelacionados de la tríada de la atleta femenina, un síndrome potencialmente serio que se observa frecuentemente en niñas y mujeres físicamente activas y que puede causar menoscabo en el rendimiento, morbilidad, tanto a corto como a largo plazo, e incluso mortalidad [39].

Más específicamente, los trastornos del comer se refieren a conductas en relación con la comida que utilizan las personas para perder o controlar su peso corporal o lograr un aspecto delgado o estético [39]. Se ha descrito que la prevalencia de trastornos patológicos del comer, como la anorexia nerviosa, la bulimia nerviosa y la anorexia atlética, es significativamente superior en atletas que en personas de control no activas, mayor en atletas femeninas que en atletas masculinos y más corriente en personas que compiten en deportes que dependen de la delgadez y del peso que en otros deportes [40]. En contraste, en un estudio en el que participaron 173 atletas femeninas finlandesas de diversos deportes, Fogelholm e Hiilloskorpi [41] confirmaron que, aunque el riesgo de los trastornos del comer puede depender del tipo de deporte, la hipótesis de que algunos grupos de atletas femeninas conllevan 
un mayor riesgo que los controles no ha sido verificada adecuadamente. Cada uno de estos comportamientos puede dar lugar a trastornos de la función menstrual normal. Se ha descrito una incidencia elevada de amenorrea secundaria en atletas participantes en determinados deportes; se ha demostrado que estas pautas menstruales anormales producen un efecto negativo sobre la mineralización ósea y, en consecuencia, pueden tener consecuencias serias, a corto y a largo plazo, incluyendo un aumento de la incidencia de fracturas por sobrecarga y osteoporosis [39, 42, 43]. La malnutrición ha sido asocia$\mathrm{da}$ a retrasos en el crecimiento y la maduración sexual, e incluso a la infertilidad [38, 42, 44].

En un estudio epidemiológico exhaustivo, Torstveit y Sundgot-Borgen [45] investigaron el porcentaje de atletas de elite femeninas y controles a riesgo de desarrollar la tríada de la atleta femenina. Una cohorte considerable de atletas y controles ajenos a este tipo de actividades completaron un cuestionario detallado, en el que se incluían preguntas referentes a pautas de entrenamiento y/o actividades físicas, historia menstrual, uso de anticonceptivos orales, historia del peso, patrones de consumo, historia nutricional y las subescalas de Insatisfacción con el Cuerpo e Impulso a la Delgadez, del Inventario de Trastornos del Comer. La batería de cuestionarios se aplicó a la población total de atletas femeninas de elite de Noruega, representativas de los equipos nacionales juveniles y veteranos (intervalo de edades: 13 a 39 años; $\mathrm{n}=669$ ) y a un grupo de controles de edades equiparables $(n=607)$. El estudio reveló resultados interesantes: (a) un mayor porcentaje de controles $(69,2 \%)$ que de atletas $(60,4 \%)$ fue considerado portador de riesgo de presentar la tríada de la atleta femenina ( $\mathrm{p} \leq 0,01$ ); (b) igualmente, un mayor porcentaje de controles informó del empleo de métodos patológicos para controlar el peso; (c) los controles presentaban mayores puntuaciones de Insatisfacción con el Cuerpo ( $\mathrm{p} \leq 0,001$ ). Sin embargo, (a) más atletas notificaron disfunción menstrual y fractura por sobrecarga en comparación con los controles ( $\mathrm{p} \leq 0,05$ ), y (b) el porcentaje de atletas que competían en deportes de delgadez $(70,1 \%)$ y el porcentaje de controles $(69,2 \%)$, consideradas de riesgo de presentar la tríada femenina, fueron superiores en comparación con las atletas que competían en deportes no centrados en la delgadez $(55,3 \%$; $\mathrm{p} \leq 0,001)$ [45]. Además, un mayor porcentaje de atletas que competían en deportes estéticos $(66,4 \%)$ que en deportes basados en juegos de pelota $(52,6 \%)$ fue considerado con riesgo de presentar la tríada ( $\mathrm{p} \leq 0,001)$. Se llegó a la conclusión de que las atletas que competían en deportes en los que la delgadez ocupaba un lugar preferente, así como los controles ajenos a las actividades atléticas, se considera- ron con riesgo de presentar la tríada en comparación con atletas que competían en deportes ajenos a la delgadez, como los juegos de pelota [45]. Los resultados del estudio noruego confirman la hipótesis de que una proporción significativa de atletas femeninas presenta uno o más componentes de la tríada $[45,46]$. También es importante recalcar que los autores comunicaron un riesgo significativo de presentar la tríada en mujeres activas normales. En consecuencia, no sólo es necesario elaborar programas y herramientas educacionales para contribuir a prevenir la tríada, sino que estos programas tienen que orientarse a niñas y mujeres jóvenes físicamente activas [46].

\section{Irregularidades menstruales en la pubertad}

En atletas adolescentes se han observado irregularidades menstruales, que comprenden la amenorrea primaria (definida como ausencia de menstruación a los 16 años), la amenorrea secundaria (definida como ausencia de periodos durante tres o más meses en mujeres que han alcanzado la menarquia) y la oligomenorrea (definida como una duración del ciclo menstrual superior a 40 días). La prevalencia de 'amenorrea atlética' varía en las atletas adolescentes del 12 al 66\% [47, 48]. Para estimar la influencia del entrenamiento intensivo sobre los ciclos menstruales en atletas femeninas, Dusek [12] examinó el momento de la menarquia y la prevalencia de amenorrea primaria y secundaria y dismenorrea en 72 atletas femeninas jóvenes y activas (de 15 a 21 años de edad) y en 96 controles de edades equiparables que no participaban en ninguna actividad deportiva organizada o regular. La muestra atlética estaba formada por jugadoras de voleibol, jugadoras de baloncesto, bailarinas de ballet y corredoras. Los resultados revelaron que la prevalencia de amenorrea secundaria era tres veces superior en las atletas que en los controles ( $\mathrm{p} \leq 0,05)$. La prevalencia de amenorrea primaria fue significativamente superior en atletas que en controles ( $p \leq 0,05)$, si bien la prevalencia de dismenorrea fue dos veces inferior en las atletas que en los controles ( $p \leq 0,001)$. La prevalencia máxima de amenorrea secundaria correspondió a las corredoras, sobre todo a las corredoras de largas distancias, en tanto que se registraba un solo caso de amenorrea secundaria entre las jugadoras de baloncesto [12]. Las corredoras de largas distancias eran también más livianas y delgadas que los controles y las atletas de otros deportes $(\mathrm{p} \leq 0,05)$. En un estudio previo de Toriola [49], la función menstrual fue evaluada en diferentes categorías de 155 atletas nigerianas (de 13 a 19 años de edad) y 135 controles (de 12 a 18 años de edad). La menstruación era más regular en los contro- 
les (44\%) que en las atletas (21\%), mientras que la amenorrea secundaria o la oligomenorrea era más prevalente en las atletas (25\%) que en los controles (10\%). Independientemente de la actividad deportiva, las personas oligomenorréicas presentaban un peso corporal considerablemente menor en relación con la talla y eran más delgadas que las personas incluidas en las demás categorías menstruales [49]. En el grupo oligomenorréico, una mayor proporción de atletas (59\%) y controles (57\%) presentaba valores de masa corporal magra por debajo del percentil 41 [49]. También se comunicó que los trastornos menstruales eran relativamente más comunes en las atletas que comenzaban a entrenar antes de la menarquia $[49,50]$.

Recientemente, Torstveit y Sundgot-Borgen [10] examinaron la prevalencia de disfunción menstrual en la población total de atletas femeninas noruegas de elite $(\mathrm{n}=669)$, que representaban al país en los niveles juvenil o veterano (edades entre 13 y 39 años), y la compararon con una muestra representativa de controles nacionales de edades equiparables $(n=607)$. Se aplicó a todas las participantes un cuestionario detallado que comprendía preguntas sobre el entrenamiento y/o patrones de actividades físicas, historia menstrual, dietética y del peso, el uso de anticonceptivos orales y el inventario de trastornos del comer. Los resultados revelaron que un porcentaje mayor de atletas $(7,3 \%)$ que de controles $(2,0 \%)$ reportó una historia de amenorrea primaria ( $\mathrm{p} \leq 0,001)$. Además, un mayor porcentaje de atletas que competían en deportes de delgadez reportó disfunción menstrual actual $(24,8 \%)$ en comparación con las atletas que competían en deportes ajenos a la delgadez $(13,1 \% ; \mathrm{p} \leq 0,01)$ y con las personas de control ( $\mathrm{p} \leq 0,05)$ [10]. Estos datos incluyen prácticamente a todas las atletas de elite elegibles y, por tanto, amplían sustancialmente los estudios previos. Los autores sugirieron que la prevalencia de disfunción menstrual entre las atletas de elite y los controles no es tan importante como se había supuesto previamente, y que la prevalencia de disfunción menstrual es menor tanto en las atletas como en los controles, en comparación con observaciones efectuadas en la década precedente [10]. No obstante, un mayor porcentaje de atletas que compiten en deportes en los que destaca la delgadez y/o un peso concreto notificó la presencia de disfunción menstrual en comparación con atletas que compiten en deportes enfocados mucho menos en tales factores [10].

En estudios transversales en atletas amenorréicas, se han observado patrones anormales de hormonas reproductoras, dando a entender un trastorno de la secreción normal de GnRH por parte del hipotálamo y, en consecuencia, la aparición de un fallo en el inicio de la función hipotalámica-hipofisaria-ovárica normal, lo que ocasio-

La pubertad en atletas femeninas na una disminución de la liberación de gonadotropinas. Los niveles hormonales gonadotrópicos y ováricos en atletas amenorréicas no muestran variación mensual en las fases folicular y luteínica, es decir, signos de supresión ovárica. Las concentraciones de estradiol y progesterona en la fase luteínica de la única gimnasta menárquica del estudio de Klentrou et al. [33] fueron menores que las concentraciones determinadas en los controles. Por otra parte, los niveles de $17 \beta$-estradiol en los controles, notificados en este estudio, no alcanzaron niveles de adulto en la fase folicular ni en la fase luteínica; sin embargo, fueron similares a los descritos previamente en adolescentes, y los niveles plasmáticos de progesterona en los controles se situaron en el mismo margen que los descritos en adultos. En estudios preliminares se comunicaron también niveles menores de estradiol y progesterona en la fase luteínica de nadadoras adolescentes en comparación con controles de edades equiparables y adultos, lo que alentó a suponer que los cuerpos lúteos de las nadadoras no estaban funcionando correctamente [51]. También se observó disfunción en la ovulación en atletas competitivas con menstruaciones regulares, cuyos resultados demostraban que los niveles de progesterona eran menores durante la fase luteínica [38]. Entre las causas sugeridas para estas alteraciones destacan las siguientes: (a) hipercortisolismo (aumento de la secreción de hormona liberadora de corticotropinas) inducido por una restricción intensa de proteínas y calorías, que causa una alteración en la pauta de secreción episódica normal de LH [47, 52]; (b) hipoestrogenismo debido a la supresión del generador de impulsos de GnRH; y (c) ausencia de un mecanismo de retroalimentación estrogénico positivo. Se ha comunicado que el mecanismo sugerido causante de irregularidades menstruales en nadadoras corresponde a un hiperandrogenismo [14].

Durante la pubertad, la maduración del eje hipotalámico-hipofisario ocasiona un incremento de la frecuencia y la amplitud de la secreción pulsátil de GnRH, cuyo resultado es un incremento de las secreciones pulsátiles de LH, FSH y esteroides sexuales. La cronología de los impulsos es esencial para establecer una ciclicidad ovulatoria normal. El pico de LH aparece por la noche durante el inicio de la pubertad y a lo largo del día y la noche, con una amplitud menor por la noche, durante la pubertad tardía y la edad adulta [53]. En los años menárquicos iniciales, la retroalimentación hipotalámica-hipofisariaovárica es inestable debido a un incremento del LH basal y a una cronología 'inmadura' de las ondas de impulsos de LH. La consecuencia consiste en ciclos menstruales irregulares, hiperandrogenismo ovárico, multifolicularidad y anovulación [54]. Por lo tanto, los ciclos infrecuen- 
tes, irregulares y anovulatorios, la hemorragia uterina anómala y la dismenorrea son corrientes durante los primeros años postmenárquicos [2, 3]. En el curso del desarrollo puberal normal, un ciclo puede considerarse normal si su duración es de 21 a 40 días, la pérdida de sangre de 20 a $80 \mathrm{ml}$ y la duración de las menstruaciones de 2 a 8 días [2,3]. La etapa final de maduración es la aparición de una retroalimentación estrogénica positiva en la hipófisis y el hipotálamo, que estimula la onda de impulso de LH en el medio del ciclo, necesaria para la ovulación. La consecuencia de la ausencia de retroalimentación estrogénica positiva en el primer año tras la menarquia cosiste en ciclos anovulatorios, pudiendo reflejar también una etapa transitoria normal en el hiperandrogenismo ovárico [3]. Por otra parte, los ciclos ovulatorios adolescentes se caracterizan por una fase folicular larga, una ovulación tardía y una fase luteínica corta e insuficiente. La selección de un folículo dominante es irregular y el folículo desarrollado presenta un diámetro menor que en la edad adulta [55]. Todas estas irregularidades suelen resolverse con el inicio de la ovulación regular [2,3]. Por lo tanto, la amenorrea en las atletas puede representar simplemente un signo de pubertad tardía o un problema inducido por la restricción de calorías, que afecta al eje hipotalámicohipofisario-ovárico.

\section{Papel de la restricción calórica}

En el contexto de este material, los trastornos del comer pueden ser utilizados como sinónimo del estado de nutrición baja. Tal como se ha mencionado anteriormente, un temor importante con respecto a la salud de atletas femeninas, jóvenes y de alto rendimiento estriba en que su ingesta calórica sea insuficiente para la cantidad de energía consumida durante el entrenamiento diario. La combinación de ejercicio intensivo y el estado de nutrición baja produce un efecto intenso sobre la liberación de las gonadotropinas, la activación del eje gonadal, la cronología de la pubertad y el inicio de trastornos menstruales en atletas adolescentes [32, 44]. Además, formas menos graves de restricción alimentaria, en relación con el gasto de energía, han sido relacionadas con fases luteínicas inadecuadas y/o anovulación, así como con ondas de impulsos de LH y FSH anormales durante el ciclo menstrual [38].

El proceso normal del ciclo menstrual es regulado por las interacciones complejas de las hormonas ováricas. Los niveles de varias de estas hormonas se alteran por el ejercicio intensivo. Estas alteraciones pueden repercutir también en la utilización de sustratos durante el ejercicio.

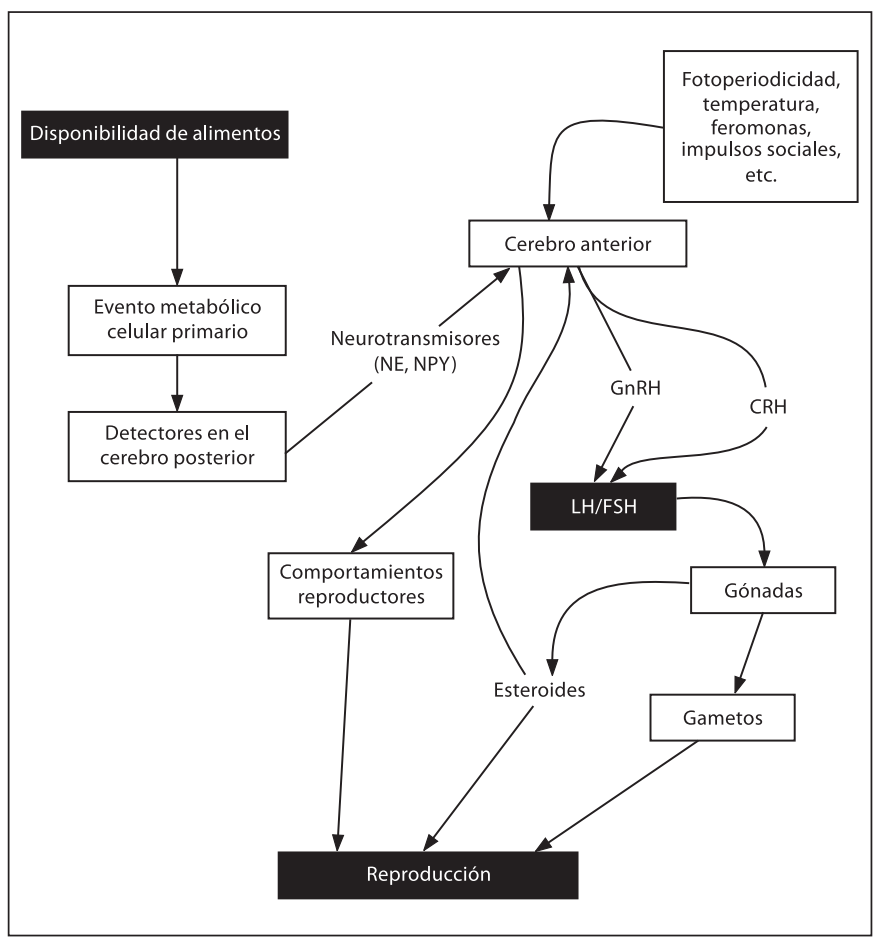

Fig. 1. Presentación esquemática de los controles nutricionales de la fisiología reproductora en mujeres. Modificado de Wade y Jones [60].

Zderic et al. [56] señalan que, en una atleta femenina correctamente nutrida, el estradiol actúa como mediador de los cambios en el metabolismo de los hidratos de carbono y las grasas. En consecuencia, la oxidación de los hidratos de carbono y las grasas puede ser regulada de manera diferente en todo el ciclo menstrual debido a los considerables cambios en las hormonas ováricas circulantes. Cuando el estradiol se eleva en la fase luteínica, la producción y la concentración de ácido láctico disminuye debido a la mayor dependencia de la oxidación de las grasas. Las mujeres presentan una mayor dependencia de la grasa como combustible cuando se reduce el lactato, independientemente de la intensidad del ejercicio, lo que permite suponer que la utilización de los lípidos puede representar un efecto directo de la elevación del estradiol [5]. Si bien existe una asociación directa, evidente, entre la utilización de combustibles y la función reproductora, persiste la duda de si también existe interacción entre la disponibilidad de los combustibles y la reproducción.

Tal como se ha mencionado previamente, se ha afirmado que las hembras deben alcanzar un nivel mínimo de grasa corporal con objeto de progresar durante la pubertad y mantener un ciclo normal [24]. Esta 'hipótesis del papel crítico de la grasa' ha sido expuesta en forma 
modificada en numerosas instancias, y ha sido aceptada por los médicos. No obstante, de acuerdo con trabajos nuevos, en realidad no existe una asociación directa de causa y efecto entre la obesidad y la función reproductora $\mathrm{y}$, aunque tienden a correlacionarse entre si, la grasa corporal y la amenorrea no han sido relacionadas de un modo causal [57-60]. Por el contrario, se ha elaborado una nueva teoría, la hipótesis de los combustibles metabólicos, que afirma que la disponibilidad de energía y no la grasa corporal regula la función reproductora en las hembras. En resumen, esta hipótesis da a entender que la función reproductora, lo mismo que la ingestión de alimentos, responde a los cambios a corto plazo en la oxidación de combustibles metabólicos. La secreción de LH y, por inferencia, de $\mathrm{GnRH}$, responde casi instantáneamente a cambios bruscos en la oxidación de los combustibles metabólicos [61]. Parece que detectores de combustibles en el cerebro posterior participan en el control de la liberación de LH. Después de que el cerebro posterior visceral detecta la información referente a la disponibilidad de combustibles, emite proyecciones directas e indirectas a las regiones del cerebro anterior, conocidas por desempeñar papeles importantes en la regulación del balance energético y la función reproductora (fig. 1). Se ha identificado que en esta vía participan proyecciones que contienen catecolaminas y neuropéptido Y [60]. Además, las proyecciones de catecolaminas y neuropéptido $\mathrm{Y}$ del cerebro anterior pueden también estimular la secreción de hormona liberadora de corticotropinas, dando lugar a hipercortisolismo que, tal como se ha dado a entender previamente, desempeña un papel predominante en la supresión de las ondas de impulsos de LH durante la subnutrición en atletas femeninas jóvenes $[47,52]$. No obstante, será imprescindible la realización de una investigación significativamente más amplia para investigar más a fondo esta interacción entre los cambios a corto plazo en la disponibilidad de combustibles y la amenorrea atlética, especialmente la que afecta a la pubertad.

\section{Conclusiones}

En el deporte, el inicio de un entrenamiento físico intensivo a una edad muy temprana causa un estrés significativo sobre el organismo durante un periodo de desarrollo crítico, especialmente en las niñas. La combinación de ejercicio intensivo y malnutrición puede producir un efecto sobre el inicio de la pubertad y la maduración sexual en atletas femeninas, especialmente en las que participan en deportes en los cuales el control del peso (es decir, aquellas que deben alcanzar una categoría de peso o incrementar al máximo el cociente entre su potencia y su peso) o el aspecto estético es una estrategia de rendimiento integrado o un componente crucial del rendimiento satisfactorio.

Si bien en algunos estudios se ha dado a entender que el retraso de la menarquia puede deberse a factores genéticos, se dispone de un conjunto considerable de pruebas a favor de que los factores ambientales desempeñan también un papel significativo. Teniendo en cuenta la constatación de que numerosos factores influyen sobre la menarquia, el papel desempeñado por el mero entrenamiento físico como factor causal en el inicio más tardío de la pubertad en niñas activas sigue siendo dudoso. Se ha descrito una mayor prevalencia de disfunción menstrual en atletas adolescentes que participan en deportes que dependen de la delgadez y del peso en comparación con las que practican otros deportes. La investigación disponible respalda el papel de factores relacionados con la nutrición y el balance energético, así como la cronología del inicio del entrenamiento intensivo, en la etiología de las irregularidades menstruales asociadas al ejercicio en atletas puberales. La naturaleza de la investigación ulterior debe ser longitudinal, con objeto de investigar los efectos a largo plazo del entrenamiento deportivo y examinar el papel que desempeñan la disponibilidad y la utilización de combustibles sobre el crecimiento y la maduración de las niñas.

\section{Bibliografía}

1 Baxter-Jones ADG, Maffulli N: Intensive training in elite young female athletes. Br J Sports Med 2002;36:13-15.

$\checkmark 2$ Caufriez A: Menstrual disorders in adolescence: pathophysiology and treatment. Horm Res 1991;36:156-159.

3 Hamm T: Physiology of normal female bleeding. NAACOGS Clin Issu Perinat Womens Health Nurs 1991;2:289-293.

La pubertad en atletas femeninas
4 Lindholm C, Hagenfeldt K, Rangertz B: Pubertal development in elite juvenile gymnasts. Effects of physical training. Acta Obstet Gynecol Scand 1994;73:269-273.

5 Bunt JC: Metabolic actions of estradiol: significance for acute and chronic exercise responses. Med Sci Sports Exerc 1990;22:286290.

6 Mansfield M, Emans J: Growth in female gymnasts: should training decrease during puberty? J. Pediatr 1993;122:237-240.
7 Theintz GE: Endocrine adaptation to intensive physical training during growth. Clin Endocrinol 1994;41:267-272.

8 Theintz GE, Ladame F, Howald $\mathrm{H}$, et al: L'enfant, la croissance et le sport de haut niveau. Schweiz Z Med Traumatol 1994;3:715.

$\checkmark 9$ Thomis M, Claessens AL, Lefevre J, Philippaerts R, Beunen GP, Malina RM: Adolescent growth spurts in female gymnasts. J Pediatr 2005;146:239-244. 
10 Torstveit MK, Sundgot-Borgen J: Participation in leanness sports but not training volume is associated with menstrual dysfunction: a national survey of 1276 elite athletes and controls. Br J Sports Med 2005;39:141147.

11 Klentrou P, Plyley M: Onset of puberty, menstrual frequency, and body fat in elite rhythmic gymnasts compared with normal controls. Br J Sports Med 2003;37:490-494.

12 Dusek T: Influence of high intensity training on menstrual cycle disorders in athletes. Croat Med J 2001;42:79-82.

13 Pigeon P, Oliver I, Charlet JP, Rochiccioli P. Intensive dance practice: repercussions on growth and puberty. Am J Sports Med 1997; 25:243-247.

14 Constantini NW, Warren MP: Menstrual dysfunction in swimmers: a distinct entity. J Clin Endocrinol Metab 1995;80:2740-2744.

15 Malina RM, Ryan RC, Bonci CM: Age at menarche in athletes and their mothers and sisters. Ann Hum Biol 1994;21:417-422.

16 Hata E, Aoki K: Age at menarche and selected menstrual characteristics in young Japanese athletes. Res Q Exerc Sport 1990;61:178-183.

17 Calabrese LH: Nutritional and medical aspects of gymnastics. Clin Sports Med 1985;4: 23-30.

18 Calabrese LH, Kirkendall DT, Floyd M: Menstrual abnormalities, nutritional patterns, and body composition in female classical ballet dancers. Phys Sportsmed 1983;11:86-98.

19 Malina R: Menarche in athletes: a synthesis and hypothesis. Ann Hum Biol 1973;10:1-24.

20 Baxter-Jones ADG, Helms PJ: Effects of train ing at a young age: a review of the training of young athletes (TOYA) study. Pediatr Exerc Sci 1996;8:310-327.

21 Kaprio J, Rimpela A, Winter T, Viken RJ, Rimpela M, Rose RJ: Common genetic influences on BMI and age at menarche. Hum Biol 1995; 67:739-753.

22 Malina RM, Waynarowska B, Bielicki T, et al: Prospective and retrospective longitudinal studies of the growth, maturation and fitness of Polish youth active in sports. Int J Sports Med 1997;18(suppl):S179-S185.

-23 Frisch R, Gotz-Welbergen AV, McArthur JW: Delayed menarche and amenorrhea of college athletes in relation to age of onset of training. JAMA 1981;246:1559-1563.

24 Frisch RE, McArthur JW: Menstrual cycles: Fatness as a determinant of minimum weight for height necessary for their maintenance or onset. Science 1974;185:949-951.

25 Bass S, Pearce G, Bradney M, et al: Exercise before puberty may confer residual benefits in bone density in adulthood: studies in active prepubertal and retired female gymnasts. J Bone Miner Res 1998;13:500-507.

26 Theintz GE, Howald H, Weiss U, et al: Evidence for a reduction of growth potential in adolescent female gymnasts. J Pediatr 1993 122:306-313.

27 Bass S, Bradney M, Pearce G, et al: Short stature and delayed puberty in gymnasts: influence of selection bias on leg length and the duration of training on trunk length. J Pediatr 200;136:149-155.
28 Ledoux M, Brisson G, Peronnet F: Nutritional habits of young female gymnasts. Med Sci Sports Exerc 1983;14:145.

29 Ersoy G: Dietary status and anthropometric assessment of child gymnasts. J Sports Med Phys Fitness 1991;31:577-580.

30 Benardot D, Czerwinski C: Selected body composition and growth measures of junior elite gymnasts. J Am Diet Assoc 1991;91:2933.

31 Damsgaard R, Bencke J, Matthiesen G, et al: Is pubertal growth adversely affected by sport? Med Sci Sports Exerc 2000;32:1698-1703.

32 Warren MP: The effects of exercise on pubertal progression and reproductive function in girls. J Clin Endocrinol Metab 1980;51:11501157.

33 Klentrou P, Flouris AD, Plyley M: Pubertal maturation, hormonal levels and body composition in Canadian male and female gymnasts; in Marfell-Jones M, Stewart A, Gordon R, Olds T (eds): Kinanthropometry IX. Proc 9th Int Conf International Society for the Advancement of Kinanthropometry (ISAK). London, Routledge, 2006, in press.

34 Malina RM, Waneen W, Spirduso CT, Baylor A: Age at menarche and selected menstrual characteristics in athletes at different competitive levels and in different sports. Med Sci Sports 1978;10:218-222.

35 Peltenburg AL, Erich WBM, Thijssen JJH, et al: Sex hormone profiles of premenarcheal athletes. Eur J Appl Physiol 1984;52:385-392.

36 Jaffré C, Lac G, Benhamou CL, Courteix D: Effects of chronic intensive training on androgenic and cortisol profiles in premenarcheal female gymnasts. Eur J Appl Physiol 2002;87:85-89.

37 Wilmore JH: Body weight standards and athletic performance; in Brownell KD, Rodin J, Wilmore JH (eds): Eating, Body Weight and Performance in Athletes: Disorders of Modern Society. Philadelphia, Lea \& Febiger, 1992, pp 315-329.

38 Loucks AB, Vaitukaitis J, Cameron JL, et al: The reproductive system and exercise in women. Med Sci Sports Exerc 1992;24 (suppl): S288-S293.

39 Yeager KK, Agostini R, Nattiv A, et al: The female athlete triad. Med Sci Sports Exerc 1993;25:775-777.

40 Sundgot-Borgen J, Torstveit MK: Prevalence of eating disorders in elite athletes is higher than in the general population. Clin J Sport Med 2004;14:25-32.

41 Fogelholm M, Hiilloskorpi H: Weight and diet concerns in Finnish female and male athletes. Med Sci Sports Exerc 1999;31:229-235.

42 Drinkwater BL, Nilson K, Chesnut CH, et al: Bone mineral content of amenorrheic and eumenorrheic athletes. N Engl J Med 1984;311: 277-281.

43 DiFiori JP: Menstrual dysfunction in athletes. How to identify and treat patients at risk for skeletal injury. Postgrad Med 1995;97:143156.
44 Loucks AB, Mortola JF, Girton L, et al: Alterations in the hypothalamic-pituitary-ovarian and the hypothalamic-pituitary-adrenal axes in athletic women. J Clin Endocrinol Metab 1989;68:402-411

45 Torstveit MK, Sundgot-Borgen J: The female athlete triad: are elite athletes at increased risk? Med Sci Sports Exerc 2005;37:184-193.

46 Torstveit MK, Sundgot-Borgen J: The female athlete triad exists in both elite athletes and controls. Med Sci Sports Exerc 2005;37:14491459.

47 Constantini NW, Warren MP: Special problems of the female athlete. Baillieres Clin Rheumatol 1994;8:199-219.

48 Patterson DF: Menstrual dysfunction in athletes: assessment and treatment. Pediatr Nurs 1995:21:227-229, 310

49 Toriola AL: Survey of menstrual function in young Nigerian athletes. Int J Sports Med 1988;9:29-34.

50 Toriola AL, Mathur DN: Menstrual dysfunction in Nigerian athletes. Br J Obstet Gynaecol 1986;93:979-985

51 Bonen A, Belcastro AN, Ling WY, et al: Profiles of selected hormones during menstrual cycles of teenage athletes. J Appl Physiol 1981; 50:545-551.

52 Fichter MM, Pirke KM: Effect of experimental and pathological weight loss upon the hypothalamo-pituitary-adrenal axis. Psychoneuroendocrinology 1986;11:295-305.

53 Wenninck JMB, Delemarre-Van De Waal HA Shoemaker R, Shoemaker H, Shoemaker I: Luteinizing hormone and follicle stimulating hormone secretion patterns in girls throughout puberty measured using highly sensitive immunoradiometric assays. Clin Endocrinol 1990;33:333-344

54 Venturoli S, Porcu E, Fabbri R, et al: Ovarian multifollicularity, high LH and androgen plasma levels, and anovulation are frequent and strongly linked in adolescent irregular cycles. Acta Endocrinol 1986;111:368-372.

55 Apter D, Räisänen I, Ylöstalo PRV: Follicular growth in relation to serum hormonal patterns in adolescent compared with adult menstrual cycle. Fertil Steril 1987;47:82-88

56 Zderic TW, Coggan AR, Ruby BC: Glucose kinetics and substrate oxidation during exercise in the follicular and luteal phases. J Appl Physiol 2001;90:447-453.

57 Bronson FH, Manning JM: The energetic regulation of ovulation: a realistic role for body fat. Biol Reprod 1991;44:945-950.

58 Loucks AB, Verdun M, Heath EM: Low energy availability, not stress of exercise, alters LH pulsatility in exercising women. J Clin Endocrinol Metab 1998;84:37-46.

59 Loucks AB: Energy availability, not body fatness, regulates reproductive function in women. Exerc Sports Sci Rev 2003;31:144-148.

60 Wade GE, Jones JE: Lessons from experimental disruption of estrous cycles and behaviors. Med Sci Sports Exerc 2003:35:1573-1580.

61 Schneider JE, Wade GN: Inhibition of reproduction in service of energy balance; in Wallen K, Schneider JE (eds): Reproduction in Context. Cambridge, MIT Press, 1999, pp 35 82. 